\title{
Prevalence of probable obstructive sleep apnea risk and severity in a population of dental patients
}

\author{
Daniel J. Levendowski • Todd Morgan • Jon Montague • \\ Victoria Melzer • Chris Berka • Philip R. Westbrook
}

Received: 25 January 2008 /Revised: 7 February 2008/Accepted: 17 February 2008/Published online: 9 April 2008

(C) The Author(s) 2008

\begin{abstract}
Obstructive sleep apnea is a commonly undiagnosed chronic disease. While dentists represent an important resource for identifying people at risk for primary snoring and sleep apnea, less than $50 \%$ of dentists are capable of identifying the common signs and symptoms of sleep disordered breathing. The aim of this study is to assess the prevalence of probable obstructive sleep apnea/ sleep disordered breathing and symptoms associated with this condition in a population of dental patients using a validated questionnaire and software that could be administered in a dental office. A retrospective analysis conducted at two dental practices using questionnaire responses obtained from 175 men and 156 women, and sleep study data obtained in the patient's homes from 75 men and 30 women with a portable recorder. Forty-six percent of the men and $19 \%$ of the women reported snoring frequently or always. Of the $67 \%$ of the men and $28 \%$ of the women identified as having a high pre-test probability (high risk) of having at least mild sleep apnea, over 33\% of the men and $6 \%$ of the women surveyed were predicted to have
\end{abstract}

D. J. Levendowski $(\bowtie) \cdot$ C. Berka $\cdot$ P. R. Westbrook

Advanced Brain Monitoring, Inc.,

2237 Faraday Avenue, Suite 100,

Carlsbad, CA 92008, USA

e-mail: Dan@b-alert.com

T. Morgan

Scripps Memorial Hospital,

320 Santa Fe Drive, Suite 105,

Encinitas, CA 92024, USA

J. Montague $\cdot$ V. Melzer

La Costa Dental Group,

501 North El Camino Real Suite 200,

Encinitas, CA 92024-1335, USA moderate or severe sleep apnea. In a subgroup of 105 patients classified at high risk who completed an overnight sleep study, 96\% had an apnea hypopnea index (AHI) greater than five events per hour. Seventy percent of those predicted to have moderate or severe OSA by questionnaire had an AHI greater than 20. All patients previously diagnosed with sleep apnea were correctly classified at high risk by ARES questionnaire. There was a high concordance between the predicted OSA risk and the degree of sleep disordered breathing. The high prevalence of undiagnosed sleep apnea in dental patients suggests that dentists could provide a valuable service to their patients by incorporating sleep apnea screening and treatment into their practice. Those who practice sedation dentistry should consider additional precautions when managing patients with risk of sleep apnea.

Keywords Sleep apnea $\cdot$ Sleep disorder breathing . Home monitoring $\cdot$ Prevalence $\cdot$ OSA severity $\cdot$ OSA risk

\section{Introduction}

Obstructive sleep apnea (OSA) has recently gained recognition as one of the most common, under-diagnosed chronic diseases $[1,2]$ and is responsible for more mortality and morbidity than any other sleep disorder [3]. It is characterized by frequent loud snoring and recurrent failures to breathe adequately during sleep (termed apneas or hypopneas) as a result of full or partial collapse of the upper airway. OSA causes increased accident risk because of daytime drowsiness and has been associated with hypertension, increased risk of congestive heart failure, coronary artery disease, myocardial infarction, cardiac arrhythmias, diabetes, and stroke [4-9]. 
In 1993, a large study of a middle-aged workforce estimated that $4 \%$ of men and $2 \%$ of women met the criteria for sleep apnea syndrome [i.e., Apnea Hypopnea Index (AHI) of five or more events per hour and hypersomnolence], and $24 \%$ and $9 \%$, respectively, had sleep disordered breathing (i.e., $\mathrm{AHI} \geq 5$ )[10]. More recent reports suggest that the prevalence of OSA is increasing in part due to the rise in obesity, a major risk factor for OSA. In 1993, obesity, based on a body mass index $(\mathrm{BMI}) \geq 30$, impacted $15-19 \%$ of the population in one quarter of the USA [11]. In $2006,92 \%$ of the US states reported obesity rates in excess of those considered extreme in 1993 (i.e., 15-19\%). Almost half of the US states now report at least $25 \%$ of their population is obese [11]. The prevalence of OSA has shown a corresponding increase. In St. Louis, MO, USA, where the obesity rate is $28 \%, 19 \%$ of an adult surgical population was estimated to have moderate, severe, or very severe OSA [12]. A telephone survey conducted by the National Sleep Foundation found that $31 \%$ of men and $21 \%$ of women met the Berlin questionnaire criteria, indicating a high risk of OSA [13]. In another recent study, $53 \%$ of the male transportation workers were identified as being at "high risk" of OSA with $22 \%$ predicted to have severe OSA using the ARES questionnaire [14].

The aim in this study was to assess the prevalence of probable OSA in a population of dental patients.

\section{Materials and methods}

A retrospective study design was selected to assess the prevalence of OSA in dental patients at two study sites in San Diego County, CA, USA, using the Apnea Risk Evaluation System (ARES ${ }^{\mathrm{TM}}$ ) questionnaire (Fig. 1). The ARES questionnaire is a validated one-page instrument that can be filled out by the patient in less than 5 min without assistance [14]. The ARES software (Advanced Brain Monitoring, Carlsbad, CA, USA) allows easy input of questionnaire responses using either a computer or an inexpensive desktop scanner. The results are immediately computed and written to a database, with a report prepared for printing.

The ARES analyses provides classifications of OSA risk (i.e., those in need of a sleep study), which includes "high risk" [i.e., predicted apnea/ hypopnea index (AHI) five or more events per hour), "low risk" (i.e., not classified as high-risk but indications such as co-morbid disease, high BMI or neck circumference plus hypersomnolence, etc.), or "no apparent risk" $(\mathrm{AHI}<5)$. The OSA severity categories include minimal (predicted $\mathrm{AHI}<5$ ), mild $(\mathrm{AHI}=6-20)$, moderate $(\mathrm{AHI}=21-40)$, and severe $(\mathrm{AHI}>40)$.

The first dental practice had no prior experience treating OSA or fitting mandibular repositioning devices (MRD). Two-hundred and twenty-nine consecutive ARES question- naire responses were obtained from patients upon arrival for a scheduled dental appointment.

The second dental office had been fitting MRDs for the treatment of OSA for over 15 years. For this practice, questionnaires were mailed to 870 patients between the ages of 45 and 80 years and who were not being treated by the dentist for OSA. Of the 108 returned responses, 102 were complete and could be used for analysis $(12.4 \%$ response rate).

A subgroup of 55 patients from the first dental office and 50 patients from the second dental office who were identified high-risk by questionnaire (total of 75 men and 30 women) completed a two-night overnight sleep study with the ARES Unicorder (Advanced Brain Monitoring) $[15,16]$. From a single site on the forehead, the wireless unicorder recorded oxygen saturation and pulse rate (reflectance pulse oximetry), airflow (by nasal a cannula connected to a pressure transducer), respiratory effort (a combined signal using pressure transducer sensing forehead venous pressure, venous volume by photoplethysmography, and actigraphy), snoring levels (calibrated acoustic microphone), head movement, and head position (accelerometers; Fig. 2). These algorithms identify apneas based on a 10-s cessation in airflow. Hypopneas require a $50 \%$ change in airflow plus a minimum desaturation and resaturation, and at least one surrogate arousal indicator (defined behaviorally as brief changes in snoring patterns, pulse rate, and/or head movement). The minimum required desaturation/ resaturation depends on the $\mathrm{SpO} 2$ level at the beginning of the OSA event (i.e., a $1 \%$ desaturation/resaturation is required if the $\mathrm{SpO} 2$ at the beginning of the event is $>93 \%$, a $1.2 \%$ desaturation/resaturation when the baseline $\mathrm{SpO} 2$ is between $93 \%$ and $91 \%$, and $3.5 \%$ desaturation and $2.5 \%$ resaturation when the baseline $\mathrm{SpO} 2$ is below 91\%). Surrogate arousal indicators must occur within the arousal window, i.e., beginning at the nadir of the airflow event and ending at the point of maximum airflow after the obstructive breathing. The patterns used to detect surrogate arousal indicators include changes in snoring patterns [e.g., crescendo/decrescendo, absence (resumption) across the nadir of the flow event, etc.], increase or decrease in pulse rate of six beats per minute, and abrupt changes in actigraphy A full description of the unicorder and the automated scoring algorithms applied to the acquired signals to characterize an apnea/hypopnea index criteria have been previously described $[15,16]$. The ARES is Food and Drug Administration-cleared for use in the USA and is CE-marked and cleared for use in European communities and Canada. Studies with a minimum of $5 \mathrm{~h}$ of valid recording time were included in the analysis.

$T$ tests and chi-squared analysis were used to assess differences in the demographics and prevalence between the two dental practices. 
ARES $^{\mathrm{TM}}$ Screener Questionnaire $\odot$

PRINT IN CAPITAL LETTERS - STAY WITHIN THE BOX

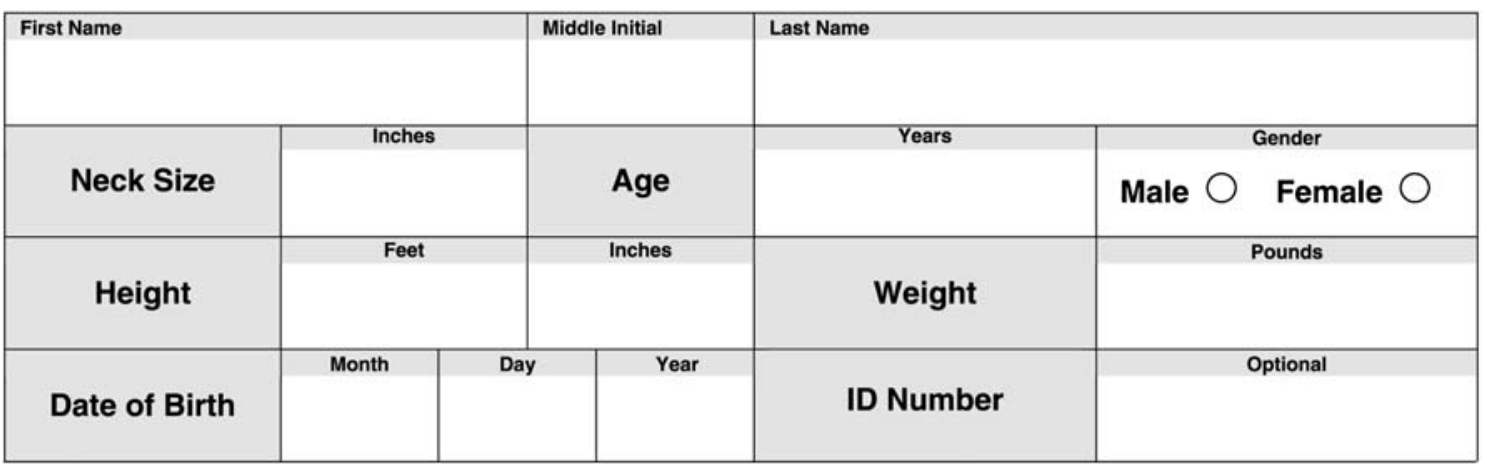

COMPLETELY FILL IN ONE CIRCLE FOR EACH QUESTION - ANSWER ALL QUESTIONS

\begin{tabular}{|lllllll}
\hline Have you been diagnosed or treated for any of the following conditions? \\
High Blood Pressure & Yes $\bigcirc$ & No $\bigcirc$ & Sleep Apnea & Yes $\bigcirc$ & No $\bigcirc$ \\
Heart Disease & Yes $\bigcirc$ & No $\bigcirc$ & Currently Pregnant & Yes $\bigcirc$ & No $\bigcirc$ \\
Diabetes & Yes $\bigcirc$ & No $\bigcirc$ & Nasal or Mask Oxygen Use & Yes $\bigcirc$ & No $\bigcirc$ \\
Stroke & Yes $\bigcirc$ & No $\bigcirc$ & Depression & Yes $\bigcirc$ & No $\bigcirc$ \\
\hline
\end{tabular}

Epworth Sleepiness Scale: How likely are you to doze off or fall asleep in the following situations, in contrast to just feeling tired? This refers to your usual way of life in recent times. Even if you have not done some of these things recently, try to work out how they would have affected you. Use the following scale to mark the most appropriate box for each situation. (M.W. Johns, Sleep 1991)

$0=$ would never doze 1 = slight chance of dozing $2=$ moderate chance of dozing $3=$ high chance of dozing

\section{Sitting and reading}

Watching TV

Sitting, inactive, in a public place (theater, meeting, etc)

As a passenger in a car for an hour without a break

Lying down to rest in the afternoon when circumstances permit

Sitting and talking to someone

Sitting quietly after lunch without alcohol

In a car, while stopped for a few minutes in traffic

$\begin{array}{llll}\mathbf{0} & \mathbf{1} & \mathbf{2} & \mathbf{3} \\ \bigcirc & \bigcirc & \bigcirc & \bigcirc \\ \bigcirc & \bigcirc & \bigcirc & \bigcirc \\ \bigcirc & \bigcirc & \bigcirc & \bigcirc \\ \bigcirc & \bigcirc & \bigcirc & \bigcirc \\ \bigcirc & \bigcirc & \bigcirc & \bigcirc \\ \bigcirc & \bigcirc & \bigcirc & \bigcirc \\ \bigcirc & \bigcirc & \bigcirc & \bigcirc \\ \bigcirc & \bigcirc & \bigcirc & \bigcirc\end{array}$

\begin{tabular}{|c|c|c|c|c|c|}
\hline \multicolumn{6}{|c|}{ On average in the past month, how often have you snored or been told that you snored? } \\
\hline Never $\bigcirc$ & $\begin{array}{l}\text { Rarely } \\
0-1 \text { times/week }\end{array}$ & \multicolumn{2}{|c|}{$\begin{array}{l}\text { Sometimes } \\
1-2 \text { times/week }\end{array}$} & $\begin{array}{l}\text { Frequently } \bigcirc \\
3-4 \text { times/week }\end{array}$ & $\begin{array}{l}\text { Almost always } \\
\text { 5-7 times/week }\end{array}$ \\
\hline \multicolumn{6}{|c|}{ Do you wake up choking or gasping? } \\
\hline Never $\bigcirc$ & $\begin{array}{l}\text { Rarely } \\
0-1 \text { times/week }\end{array}$ & \multicolumn{2}{|c|}{$\begin{array}{l}\text { Sometimes } \\
1-2 \text { times/week }\end{array}$} & $\begin{array}{l}\text { Frequently } \bigcirc \\
3-4 \text { times/week }\end{array}$ & $\begin{array}{l}\text { Almost always } \\
\text { 5-7 times/week }\end{array}$ \\
\hline \multicolumn{6}{|c|}{ Have you been told that you stop breathing in your sleep or wake up choking or gasping? } \\
\hline Never $\bigcirc$ & $\begin{array}{l}\text { Rarely } \\
0-1 \text { times/week }\end{array}$ & \multicolumn{2}{|c|}{$\begin{array}{l}\text { Sometimes } \\
1-2 \text { times/week }\end{array}$} & $\begin{array}{l}\text { Frequently } \bigcirc \\
3-4 \text { times/week }\end{array}$ & $\begin{array}{l}\text { Almost always } \\
\text { 5-7 times/week }\end{array}$ \\
\hline Signature & & Month & Year & Area Code & \\
\hline
\end{tabular}

Fig. 1 ARES screening questionnaire 


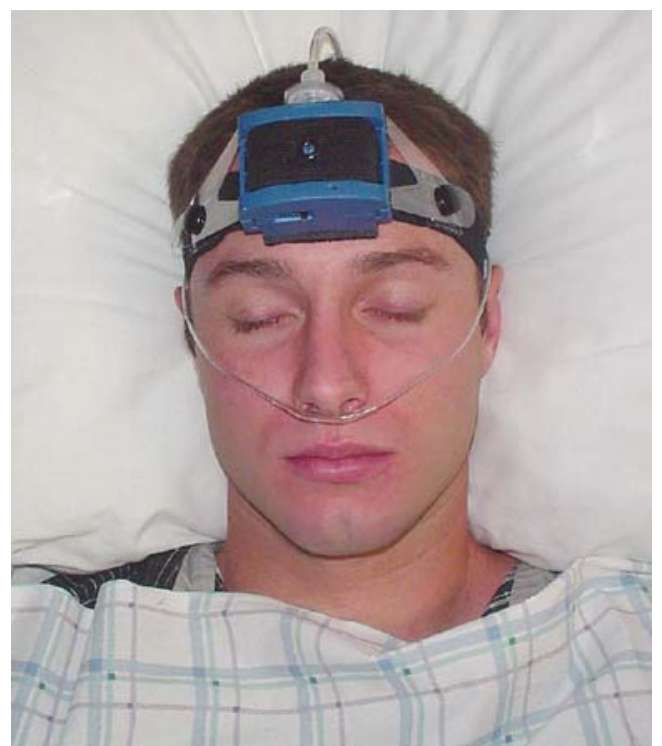

Fig. 2 Patient wearing ARES unicorder

\section{Results}

$T$ tests and chi-squared analysis confirmed that there were no significant differences in the distributions of age, BMI, neck size, Epworth sleepiness scale, and prevalence of OSA risk between the two dental offices by gender; thus, the data were combined for further analysis. Demographic and anthropomorphic characteristics of the men and women by and across practices are presented in Table 1 .

The average BMI across all participants was relatively low with only $19 \%$ of those surveyed reporting a BMI in the obese range. An equivalent percentage of men and women reported high blood pressure $(29.1 \%$ and $25.0 \%$, respectively) when the responses from the two sites were combined. A significantly greater number of women reported high blood pressure when the responses were obtained consecutively vs. the mailed survey $(28.6 \%$ vs.
Table 2 Distribution of responses typically used to identify OSA

Never (\%) Rarely Sometimes Frequent Almost $0-1 /$ week 1-2/week 3-4/week always 5-7/
(\%)
$(\%)$
$(\%)$
week $(\%)$

\begin{tabular}{|c|c|c|c|c|c|}
\hline \multicolumn{6}{|c|}{ In the past month, snored or told you snored? } \\
\hline Male & 13.1 & 18.3 & 22.9 & 16.0 & 29.7 \\
\hline Female & 34.0 & 32.7 & 14.1 & 12.2 & 7.1 \\
\hline \multicolumn{6}{|c|}{ Do you wake up choking or gasping? } \\
\hline Male & 64.0 & 13.1 & 11.4 & 9.1 & 2.3 \\
\hline Female & 78.2 & 11.5 & 8.3 & 1.3 & 0.6 \\
\hline \multicolumn{6}{|c|}{ Told you stop breathing or wake up choking or gasping? } \\
\hline Male & 62.9 & 10.9 & 10.3 & 13.7 & 2.3 \\
\hline Female & 84.6 & 7.7 & 5.8 & 1.9 & 0.0 \\
\hline
\end{tabular}

17.6\%). All 24 of the dental patients previously diagnosed with OSA were correctly classified with as high risk of OSA and $75 \%$ were predicted to have moderate or severe OSA.

Sixty-nine percent of the men and $33 \%$ of the women reported snoring at least sometimes, and $46 \%$ of the men and $19 \%$ of the women snored frequently or always (Table 2). In addition, $23 \%$ of the men and $10 \%$ of the women reported being aware of waking up choking at least sometimes, while $26 \%$ and $8 \%$ were told by bed partners that they stopped breathing or awoke choking at least sometimes.

In this dental population, $67 \%$ of the men and $28 \%$ of the women were predicted to be at high risk of having at least mild OSA (Fig. 3), while $33 \%$ and $6 \%$, respectively, were predicted to have moderate or severe OSA (Table 3).

The AHI distributions from the in-home sleep studies for the patients classified as high risk for OSA are presented in Fig. 4. Of these 105 patients, $96 \%$ had an AHI greater than five, suggesting some level of sleep disordered breathing,

Table 1 Demographic and anthropomorphic characteristics

\begin{tabular}{|c|c|c|c|c|c|c|}
\hline & \multicolumn{2}{|c|}{ Site $1-$ consecutive } & \multicolumn{2}{|c|}{ Site 2 -mailed survey } & \multicolumn{2}{|l|}{ Combined } \\
\hline & Male $(n=124)$ & Female $(n=105)$ & Male $(n=51)$ & Female $(n=51)$ & Male $(n=175)$ & Female $(n=156)$ \\
\hline \multicolumn{7}{|l|}{ Mean \pm SD } \\
\hline Age-years & $52 \pm 14.0$ & $55 \pm 15.8$ & $55 \pm 9.2$ & $56 \pm 7.7$ & $53 \pm 12.8$ & $55 \pm 13.7$ \\
\hline Neck size $-\mathrm{cm}$ & $42 \pm 2.7$ & $35 \pm 3.3$ & $42 \pm 2.7$ & $34 \pm 3.6$ & $42 \pm 2.7$ & $35 \pm 3.4$ \\
\hline Body mass index & $28 \pm 4.6$ & $26 \pm 6.1$ & $27 \pm 3.6$ & $23 \pm 4.9$ & $28 \pm 4.3$ & $25 \pm 5.8$ \\
\hline Epworth score & $7 \pm 5.1$ & $6 \pm 4.1$ & $7 \pm 4.8$ & $6 \pm 4.5$ & $7 \pm 5.0$ & $6 \pm 4.2$ \\
\hline \multicolumn{7}{|l|}{ Number $(\%)$} \\
\hline High blood pressure & $35(28.2)$ & $30(28.6)$ & $16(31.4)$ & $9(17.6)$ & $51(29.1)$ & $39(25.0)$ \\
\hline Heart disease & $11(8.9)$ & $6(5.7)$ & $4(7.8)$ & $1(2.0)$ & $15(8.6)$ & $7(4.5)$ \\
\hline Diabetes & $7(5.6)$ & $1(1.0)$ & $6(11.8)$ & $2(3.9)$ & $13(7.4)$ & $3(1.9)$ \\
\hline Stroke & $2(1.6)$ & $5(4.8)$ & $0(0.0)$ & $1(2.0)$ & $2(1.1)$ & $6(3.8)$ \\
\hline Obstructive sleep apnea & $11(8.9)$ & $2(1.9)$ & $8(15.7)$ & $3(5.9)$ & $19(10.9)$ & $5(3.2)$ \\
\hline
\end{tabular}




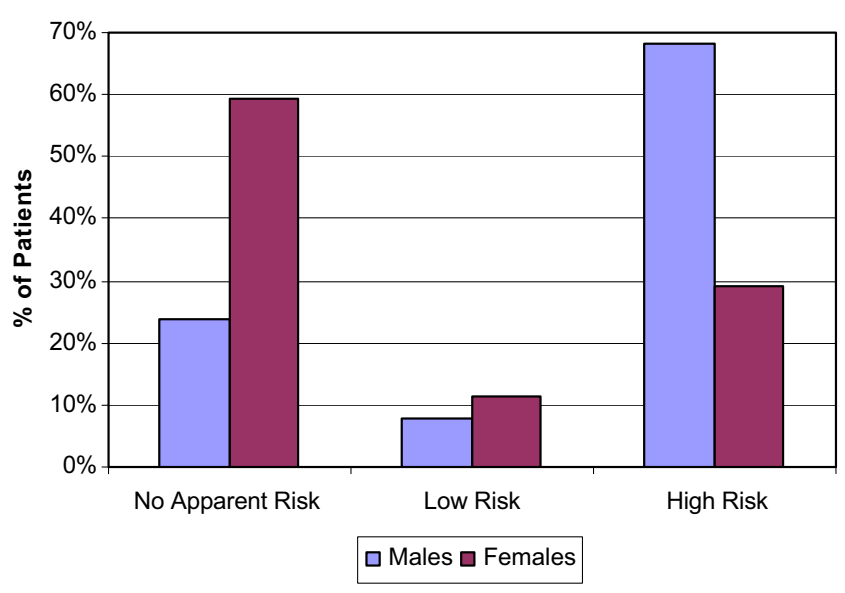

Fig. 3 Distribution of dental patients classified at risk of having OSA by questionnaire $(n=331)$

and $84 \%$ and $70 \%$ had AHIs greater than 10 and 15 , respectively.

A comparison between the predicted OSA severity by questionnaire and the degree of sleep-disordered breathing measured with an in-home sleep study is presented in Fig. 5. Of interest, $70 \%(41: 59)$ of those predicted to have moderate or severe OSA had an AHI $>20$.

\section{Discussion}

Possibly, as a result of the age of the surveyed population, only $25 \%$ of the men and $60 \%$ of the women had no apparent risk of having OSA (Fig. 3), while $34 \%$ and $6 \%$, respectively, were predicted to have moderate or severe OSA (Table 3). Of those who completed a sleep study, $96 \%$ were confirmed to have at least mild sleep-disordered breathing (i.e., AHI $>5$ ). The term high risk was used to characterize patients who should be considered for a sleep study; however, an asymptomatic patient with an AHI of

Table 3 Distribution of predicted OSA severity stratified by OSA Risk for all dental patients

\begin{tabular}{lcccc}
\hline \multicolumn{5}{c}{ OSA severity } \\
\cline { 2 - 5 } & Minimal (\%) & Mild (\%) & Moderate (\%) & Severe (\%) \\
\hline No apparent risk & & & \\
Male & 24.6 & 0.0 & 0.0 & 0.0 \\
Female & 60.3 & 0.0 & 0.0 & 0.0 \\
Low risk & & & & \\
Male & 1.1 & 6.9 & 0.0 & 0.0 \\
Female & 3.2 & 8.3 & 0.0 & 0.0 \\
High risk & & & & \\
Male & 0.6 & 33.1 & 10.3 & 23.4 \\
Female & 3.2 & 18.6 & 5.1 & 1.3 \\
\hline
\end{tabular}

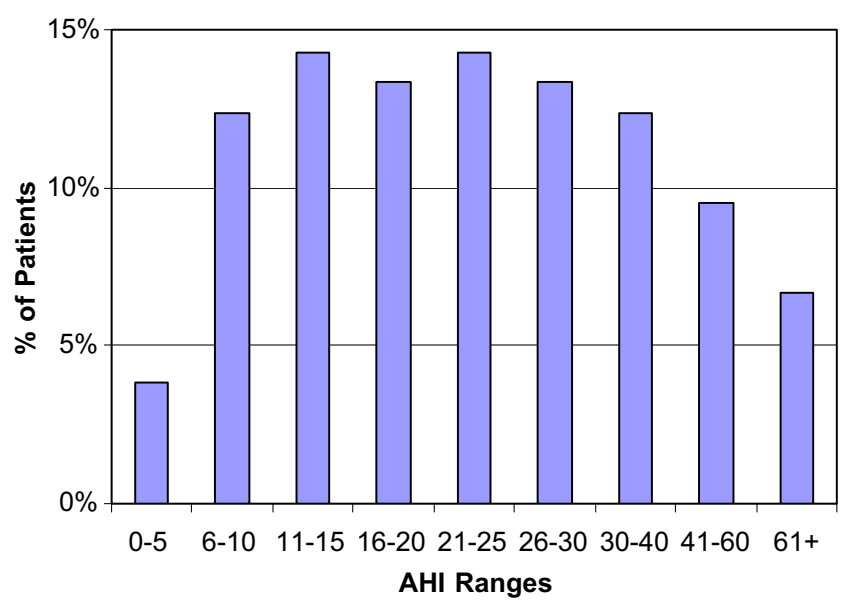

Fig. 4 Distribution of the AHI measured during an in-home sleep study

five does not suggest a perilous condition. Of interest, $70 \%$ of the patients classified at high risk by questionnaire (and previously undiagnosed) exhibited sleep-disordered breathing of at least 15 events per hour, a threshold commonly used to refer patients for treatment of OSA.

The prevalence of high risk by the ARES questionnaire in female dental patients was similar to women between 50 and 64 years classified as OSA risk by the Berlin questionnaire in the National Sleep Foundation (NSF) survey (i.e., 29\%) [13]. Comparatively, the percentage of male dental patients classified as high-risk OSA (68\%) was almost twice the number of men between 50-64 years classified at OSA risk (37\%) in the NSF survey. The sensitivity and specificity of the two instruments are similar [14]. The prevalence of OSA risk in these dental populations was similar to that reported in preoperative patients (12). In a population of male transportation workers, those

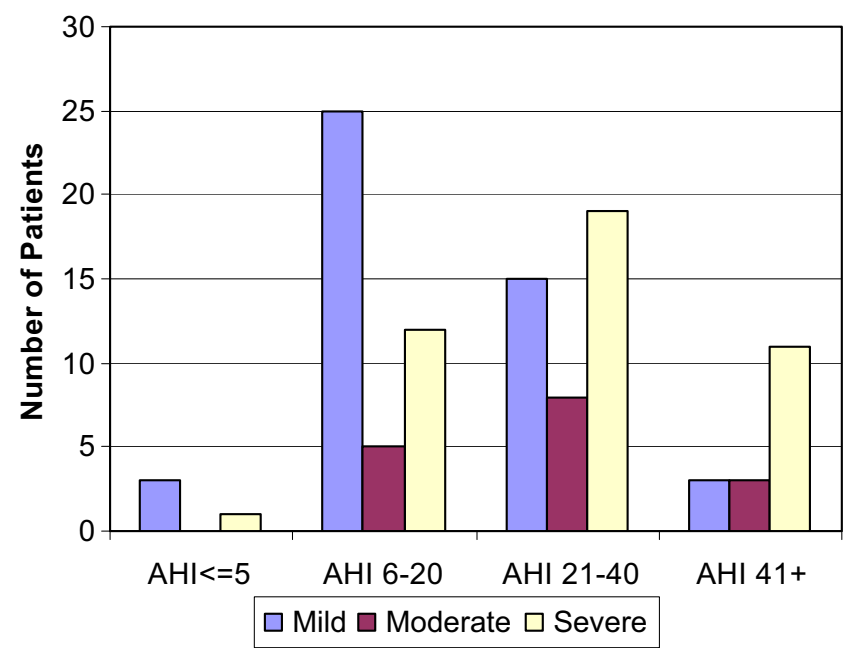

Fig. 5 Distribution of predicted OSA severity vs. the AHI measured during an in-home sleep study 
classified as high-risk of OSA by ARES questionnaire was $50 \%$, compared with $68 \%$ in male dental patients [14]. The increased prevalence in this study may be explained by differences in the mean age of the transportation workers and dental patients (i.e., 41 and 53 years, respectively), given that the risk of OSA increases with age [13].

The subgroup of patients who completed sleep studies was selected based on the need to rule out individuals for a clinical study protocol. Thus, one of the limitations of this study was that the sensitivity and specificity of the ARES questionnaire could not be cross-validated against the previous report (i.e. 0.94 and 0.76 , respectively). The positive predictive value of the instrument in this community-based population, however, was slightly better than that of the previous observation (i.e., 0.96 vs. 0.91, respectively) [14].

The proportion of patients classified with severe OSA by questionnaire that had an AHI greater than 40 was less than previously reported [14]. This is likely a result of developing the predictive model using questionnaire responses from a more select group of patients with a greater likelihood of having severe disease (e.g., suspected of having OSA or preoperative patients, etc.) vs. questionnaire responses from a community based population. It should be noted that, in both the model development data set and this community-based population, the same percentage of those with an AHI greater than 20 were predicted to have moderate or severe OSA (i.e., 70\%).

In this survey, $46 \%$ of men and $19 \%$ of women snored at least three times per week, and $37 \%$ and $15 \%$, respectively, reported witnessed apneas. Comparatively, 37\% of Hong Kong bus drivers ( $96 \%$ male) reported snoring at a similar frequency, while only $8 \%$ reported witnessed apneas [17].

The mean percentage of dental patients with high blood pressure $(27 \%)$ and heart disease $(7 \%)$ was similar to those surveyed by the NSF (29\% and $10 \%$, respectively) [13]. Nineteen percent of the dental patients in this study were considered obese (BMI $>30$ ), while the reported obesity in California is between $20 \%$ and $25 \%$. The prevalence of diabetes in this study $(5 \%)$ was less than the $11 \%$ reported in the NSF survey, possibly as a result of the lower BMIs. The mean BMI and prevalence of obesity in this population may be low in comparison to other regions of the USA as a result of the socioeconomic factors favoring these two dental practices.

One of the goals of this study was to assess whether the prevalence of OSA risk obtained by questionnaire would be impacted by the method used to obtain the responses, as either might be appropriate for surveying a dental population. These data suggest that the prevalence of OSA risk will not be significantly biased whether responses are obtained consecutively in person or from a direct mailing.

\section{Conclusions}

The percentage of dental patients found to have a high pretest probability of having undiagnosed OSA was substantially greater than previous prevalence estimates. Given the high concordance between the predicted OSA risk and the degree of sleep disordered breathing, these results suggest that dentists could provide a valuable service to their patients by incorporating sleep apnea screening and treatment into their practice. Those who practice sedation dentistry should consider additional precautions when managing patients with risk of sleep apnea.

Acknowledgment This study was funded by the National Institute of Dental and Craniofacial Research (NIDCR-DE016772). Daniel Levendowski, Chris Berka, and Philip Westbrook are employees of and shareholders in Advanced Brain Monitoring.

Open Access This article is distributed under the terms of the Creative Commons Attribution Noncommercial License which permits any noncommercial use, distribution, and reproduction in any medium, provided the original author(s) and source are credited.

\section{References}

1. Dement WC, Vaughan CC (1999) The promise of sleep: a pioneer in sleep medicine explores the vital connection between health, happiness, and a good night's sleep. Delacorte, New York

2. Kripke DF, Ancoli-Israel S, Klauber MR, Wingard DL, Mason WJ, Mullaney DJ (1997) Prevalence of sleep-disordered breathing in ages 40-64 years: a population-based survey. Sleep 20(1):65-76

3. US Department of Health and Human Services (2000) Healthy People 2010

4. Bradley TD (1992) Right and left ventricular functional impairment and sleep apnea. Clin Chest Med 13(3):459-479

5. Duchna HW, Guilleminault C, Stoohs RA, Faul JL, Moreno H Hoffman BB, Blaschke TF (2000) Vascular reactivity in obstructive sleep apnea syndrome. Am J Respir Crit Care Med 161(1):187-191

6. Guilleminault C, Connolly SJ, Winkle RA (1983) Cardiac arrhythmia and conduction disturbances during sleep in 400 patients with sleep apnea syndrome. Am J Cardiol 52(5):490-494

7. Guilleminault C, Suzuki M (1992) Sleep-related hemodynamics and hypertension with partial or complete upper airway obstruction during sleep. Sleep 15(6 Suppl):S20-S24

8. Palomaki H (1991) Snoring and the risk of ischemic brain infarction. Stroke 22(8):1021-1025

9. Partinen M, Guilleminault C (1990) Daytime sleepiness and vascular morbidity at seven-year follow-up in obstructive sleep apnea patients. Chest 97(1):27-32

10. Young T, Palta M, Dempsey J, Skatrud J, Weber S, Badr S (1993) The occurrence of sleep-disrodered breathing among middle-aged adults. NEJM 328:1230-1235

11. Center for Disease Control. U.S. obesity trends 1985-2006, www. cdc.gov/nccdphp/dnpa/obesity. Accessed 16 Nov 2007

12. Finkel KJ, Saager L, Safarzadeh E, Bottros MM, Avidan MS (2006) Obstructive sleep apnea: the silent pandemic. Anesthesiology 105:A988

13. Hiestand DM, Britz P, Goldman M, Phillips B (2006) Prevalence of symptoms and risk of sleep apnea in the us population. Results 
from the national sleep foundation sleep in America 2005 poll. Chest 130:780-786

14. Levendowski DJ, Olmstead R, Popovic D, Zavora T, Carper D, Berka C, Westbrook P (2007) Part I-Assessment of obstructive sleep apnea risk and severity in truck drivers: validation of the screening questionnaire. Sleep Diagnosis and Therapy 2(2):20-30

15. Ayappa I, Norman RG, Seelall V, Rapoport DM (2008) Validation of a self-applied unattended monitor for sleep disordered breathing. J Clin Sleep Med 4(1):26-37
16. Westbrook PR, Levendoiwski DJ, Cvetinovic M, Zavora T, Velmirovic V, Henninger D, Nicholson D (2005) Description and validation of the apnea risk evaluation system: a novel method to diagnose sleep apnea-hypopnea in the home. Chest 128:2166-2175

17. Hui DSC, Chan JKW, Ko FWS, Choy DKL, Li TST, Chan AT, Wong KK, Lai CKW (2002) Prevalence of snoring and sleepdisordered breathing in a group of commercial bus drivers in Hong Kong. Internal Medicine Journal 32(4):149-157 